\title{
Recommendations to improve the management and the prognosis of patients with heart failure
}

\author{
Carlos Escobar ${ }^{1}$, Vivencio Barrios*,2, Carolina Ortiz Cortés ${ }^{3}$, Juan Cosín Sales ${ }^{4}$, Domingo A \\ Pascual Figal ${ }^{5}$ \& Xavier Garcia-Moll Marimón ${ }^{6}$ \\ ${ }^{1}$ Department of Cardiology, University Hospital Universitario La Paz, Madrid, Spain \\ ${ }^{2}$ Department of Cardiology, University Hospital Ramón y Cajal, Madrid, Spain \\ ${ }^{3}$ Department of Cardiology, Hospital San Pedro de Alcántara, Cáceres, Spain \\ ${ }^{4}$ Department of Cardiology, Hospital Arnau de Vilanova, Valencia, Spain \\ ${ }^{5}$ Department of Cardiology, University Hospital Virgen de la Arrixaca, Murcia, Spain \\ ${ }^{6}$ Department of Cardiology, Hospital Santa Creu i Sant Pau, Barcelona, Spain \\ *Author for correspondence: vivenciobarrios@gmail.com
}

\begin{abstract}
"The aim of this questionnaire was to create a tool that in a short time period (i.e., $<5 \mathrm{~min}$ ), the physician could be aware about the HF status of the patient during the interview in order to optimize the treatment."
\end{abstract}

First draft submitted: 21 July 2018; Accepted for publication: 18 October 2019; Published online: 25 November 2019 Keywords: decompensated heart failure $\bullet$ diuretic $\bullet$ functional class $\bullet$ furosemide $\bullet$ heart failure $\bullet$ hospitalization $\bullet$
natriuretic peptide $\bullet$ questionnaire $\bullet$ stability $\bullet$ treatment

Despite current advances in the treatment of patients with heart failure (HF), mortality rates remain unacceptably high. There is an inverse relationship between New York Heart Association (NYHA) functional class and all-cause mortality. Thus, as NYHA functional class worsens, mortality rates increase [1]. Unfortunately, therapeutic inertia is common in patients with HF. One of the reasons is that there is a trend for searching only 'clinical stability' as the main goal in the management of patients with HF [2]. However, HF is a progressive entity, even in a subclinical way, and no optimization of treatment promotes HF progression. In addition, there is a significant risk of sudden cardiac death, mainly in HF patients with a less severe NYHA functional class [3]. As a result, it is necessary to promote the search of new tools that reduce the therapeutic inertia and enhance the early modifications in the treatment of patients with HF to improve cardiovascular prognosis.

The IC-BERG study was performed to ascertain the perception and the management of patients with HF by the clinical cardiologists in Spain in order to develop practical recommendations to improve the diagnostic and therapeutic approach of this population [2]. After analyzing the results of the IC-BERG study, we detected some gaps in the management of HF patients, and we developed a simple questionnaire targeting five issues in order to minimize them. The aim of this questionnaire was to create a tool that in a short time period (i.e., $<5 \mathrm{~min}$ ), the physician could be aware about the HF status of the patient during the interview in order to optimize the treatment. In this manuscript, we present these five simple questions that should be performed in routine practice to facilitate the early detection of patients at high risk of HF decompensation and to promote changes in the treatment to optimize the management of this population (Table 1).

\section{Question 1}

Although NYHA functional class is widely used in clinical practice and it has been used as an inclusion criterion in most HF clinical trials [3], the subjectivity is considerable, and this makes more difficult its applicability in routine practice. In fact, it has been reported that there are significant disparities between the NYHA functional class perceived by the patients and their physicians [4]. We consider that in addition to the evaluation of NYHA functional class, it is better to perform some specific questions about daily activities and how the capacity to develop 
Table 1. The 5 questions to be performed in clinical practice in patients with heart failure.

Question 1: daily activities
Can you walk the same
distance as in the past year?
Can you go shopping and carry
bags as in the past year?
Can you go upstairs with the
same fatigue as in the past
year?
Can you do housework as
in the past year?
HF: Heart failure.

$\begin{array}{ll}\begin{array}{l}\text { Question 2: early } \\ \text { symptoms/signs of HF } \\ \text { decompensation }\end{array} & \begin{array}{l}\text { Question 3: use of loop } \\ \text { diuretics }\end{array} \\ \begin{array}{ll}\text { Has the patient gained body } \\ \text { weight? }\end{array} & \begin{array}{l}\text { Do you need } \geq 1 \text { pill of } \\ \text { furosemide (or equivalent) to }\end{array} \\ \begin{array}{l}\text { Does the patient have (more) } \\ \text { ankle edema? }\end{array} & \begin{array}{l}\text { keep in the same functional } \\ \text { class? }\end{array} \\ \begin{array}{l}\text { Does the patient need more } \\ \text { pillows to sleep comfortably? }\end{array} & \\ & \\ & \end{array}$

Question 4: previous visits to Question 5: if NPs are monitored the Emergency department/hospital

Have you been hospitalized or Have levels of NPs significantly visited the Emergency increased since the last visit? department due to HF decompensation in the past year?

these activities has been modified in the last $6 / 12$ months, in order to identify those patients that minimize the symptoms. Some examples of these questions might be:

- Can you walk the same distance as in the past year?

- Can you go shopping and carry bags as in the past year?

- Can you go upstairs with the same fatigue as in the past year?

- Can you do housework as in the past year?

\section{Question 2}

It is important to explore in a simple way if there are initial symptoms or signs of HF decompensation. The early identification of HF decompensation allows improving the treatment and, in many cases, avoiding visits to the Emergency department or even hospital admissions [3]. Although the use of hemodynamic monitoring systems (i.e., CardioMems) may improve the management of patients with HF, they are not available for the majority of patients and other strategies are required [5,6]. In this context, our questionnaire could be a simple option. Thus, questions in this point may include:

- Has the patient gained body weight?

- Does the patient have (more) ankle edema?

- Does the patient need more pillows to sleep comfortably?

\section{Question 3}

Although loop diuretics are very effective in reducing congestive symptoms, they have not been associated with an improvement of cardiovascular prognosis in patients with HF [3]. On the other hand, those patients that require loop diuretics to maintain the euvolemic state have a worse prognosis than those patients that do not need them. Additionally, the prognosis worsens as the required dose of diuretic increases, even as early as $40 \mathrm{mg}$ daily of oral furosemide [7]. This question may be important not only after instructing the patient to modify the dose of furosemide, but also when other physician modifies the furosemide dosage but does not perform any other changes in the HF treatment. Therefore, the patient should be specifically asked about the need of taking loop diuretics and the dose:

- Do you need $\geq 1$ pill of furosemide (or equivalent) to keep in the same functional class?

\section{Question 4}

The risk of readmission after a HF hospitalization is high [8]. In addition, it is not only important to ask for hospital admissions, but also for visits to the Emergency department that require intravenous diuretics for HF stabilization. In both cases, the risk of early decompensation is high, and optimization of HF treatment is mandatory. This should be specifically asked when attending the patient:

- Have you been hospitalized or visited the Emergency department due to HF decompensation in the past year? 


\section{Question 5}

There is a direct relationship between NPs levels and the risk of hospitalization and death [3]. However, there are many factors that may impact on NP levels (i.e., age, renal insufficiency, etc.) [3]. As a result, more important than absolute values are the relative changes of NPs, so that if there is a significant relative increase of NPs, although the patient remain in the same NYHA functional class, treatment for HF should be modified adequately. Therefore, if NPs have been monitored during the follow-up, this should be specifically analyzed:

- Have the levels of NPs significantly increased since the last visit?

If the answer is no to the first question or yes to any of the other four questions, this means that HF is progressing, and a revaluation of treatment is mandatory. Although the questions are interrelated, treatment should be modified when any of them suggest HF progression. As a result, the determination of NPs is not strictly necessary to identify those patients at high risk of destabilization.

The target of HF should be to improve functional class, and not only searching stability, as this is associated with a worse prognosis $[1,2]$. In this context, our questionnaire may be helpful to identify those patients that may require an optimization of HF therapy.

In the last years, some questionnaires have been developed for assessing quality of life or changes over time in patients with HF, including the Minnesota Living with Heart Failure Questionnaire (MLHFQ), the Chronic Heart Failure Questionnaire (CHFQ), the Quality of Life Questionnaire for Severe Heart Failure (QLQ-SHF), the Kansas City Cardiomyopathy Questionnaire (KCCQ) or the Left Ventricular Dysfunction (LVD-36) questionnaire [9-11]. However, they require many minutes to be completed, limiting their use in routine practice. In addition, other initiatives to improve the adherence to guidelines, such as the 'Get with the Guidelines' program in the USA, have numerous tasks for the clinician to treat the patients with HF [12-14]. However, as the number of tasks for the clinician increases, the utility in clinical practice decreases, as it is less practical. By contrast, our questionnaire only requires few minutes to be completed, and may be very useful when the time for the visit is limited. For example, a practical case of the utility of our questionnaires is described as follows: an outpatient with systolic HF referred 'clinical stability' during the interview and HF treatment was not optimized. Two months later, the patient is hospitalized because of decompensated HF. However, the fact is that the patient had increased the dosage of furosemide some weeks before the visit to remain in the same functional class, indicating instability. With this new questionnaire, this had been easily identified.

It should be noted that our questionnaire has not been validated yet in a prospective cohort. Our aim is to implement this questionnaire in Spain and then analyze its impact on clinical practice and compare with previous studies.

In summary, the objective of our study was to develop a simple questionnaire that could detect those patients at high risk of decompensation, when the time for the visit is limited. The answers to these five simple questions can be rapidly obtained during the visit and may allow an early optimization of HF treatment, not just for searching the stabilization of the patient, but for improving the prognosis.

\section{Financial \& competing interests disclosure}

The study was promoted by the Investigation Agency of the Spanish Society of Cardiology, with an unrestricted grant of Novartis España. The authors have no other relevant affiliations or financial involvement with any organization or entity with a financial interest in or financial conflict with the subject matter or materials discussed in the manuscript apart from those disclosed.

No writing assistance was utilized in the production of this manuscript.

\section{Disclaimer}

The study was funded in a nonrestrictive manner by the pharmaceutical company Novartis. The funder did not intervene in any way in the organization, management or analysis of the data.

\section{References}

1. Muntwyler J, Abetel G, Gruner C, Follath F. One-year mortality among unselected outpatients with heart failure. Eur. Heart J. 23(23), 1861-1866 (2002).

2. Barrios V, Escobar C, Ortiz C et al. Management of heart failure in the cardiology outpatients clinic: the IC-BERG study. Rev. Clin. Esp. 2019 (2019) (in press). 
3. Ponikowski P, Voors AA, Anker SD et al. 2016 ESC Guidelines for the diagnosis and treatment of acute and chronic heart failure: the task force for the diagnosis and treatment of acute and chronic heart failure of the European Society of Cardiology (ESC) developed with the special contribution of the Heart Failure Association (HFA) of the ESC. Eur. Heart J. 37(27), 2129-2200 (2016).

4. Goode KM, Nabb S, Cleland JG, Clark AL. A comparison of patient and physician-rated New York Heart Association class in a community-based heart failure clinic. J. Card. Fail. 14(5), 379-387 (2008).

5. Adamson PB, Abraham WT, Aaron $\mathrm{M}$ et al. CHAMPION trial rationale and design: the long-term safety and clinical efficacy of a wireless pulmonary artery pressure monitoring system. J. Card. Fail. 17(1), 3-10 (2011).

6. Gronda E, Vanoli E, Zorzi A, Corrado D. CardioMEMS, the real progress in heart failure home monitoring. Heart Fail. Rev.(2019). doi: 10.1007/s10741-019-09840-y (Epub ahead of print)

7. Sargento L, Simōes AV, Longo S, Lousada N, Reis RP. Furosemide prescription during the dry state is a predictor of long-term survival of stable, optimally medicated patients with systolic heart failure. J. Cardiovasc. Pharmacol. Ther. 22(3), 256-263 (2017).

8. Santas E, Valero E, Mollar A et al. Burden of recurrent hospitalizations following an admission for acute heart failure: preserved versus reduced ejection fraction. Rev. Esp. Cardiol. (Engl Ed). 70(4), 239-246 (2017).

9. Garin O, Ferrer M, Pont A et al. Disease-specific health-related quality of life questionnaires for heart failure: a systematic review with meta-analyses. Qual. Life Res. 18(1), 71-85 (2009).

10. Garin $\mathrm{O}$, Herdman M, Vilagut $\mathrm{G}$ et al. Assessing health-related quality of life in patients with heart failure: a systematic, standardized comparison of available measures. Heart Fail. Rev. 19(3), 359-367 (2014).

11. Patel H, Ekman I, Spertus JA, Wasserman SM, Persson LO. Psychometric properties of a Swedish version of the Kansas City cardiomyopathy questionnaire in a chronic heart failure population. Eur. J. Cardiovasc. Nurs. 7(3), 214-221 (2008).

12. Kumbhani DJ, Fonarow GC, Heidenreich PA et al. Association between hospital volume, processes of care, and outcomes in patients admitted with heart failure: insights From get with the guidelines-heart failure. Circulation. 137(16), 1661-1670 (2018).

13. Ziaeian B, Hernandez AF, DeVore AD et al. Long-term outcomes for heart failure patients with and without diabetes: from the get with the guidelines-heart failure registry. Am. Heart J. 211, 1-10 (2019).

14. Suzuki S, Yoshihisa A, Sato Y et al. Clinical significance of get with the guidelines-heart failure risk score in patients with chronic heart failure after hospitalization. J. Am. Heart Assoc. 7(17), e008316 (2018). 